\title{
Metallic Cylinder Arrays As Optical Coupler For Quantum Well Infrared Photo-detector
}

\author{
J. Y. Ding ${ }^{\text {a }}$, Q. Li ${ }^{\mathrm{a}}$, B. Ni ${ }^{\mathrm{a}}$, G. H. Li ${ }^{\mathrm{a}}$, Z. F. Li ${ }^{\mathrm{a}}$, X. S. Chen ${ }^{\mathrm{a}}{ }^{*}$ and W. Lu ${ }^{\mathrm{a}, *}$ \\ ${ }^{a}$ State Key Lab for Infrared Physics, Shanghai Institute of Technical Physics, Chinese Academy of Sciences \\ 500 Yu Tian Road, Shanghai, China 200083
}

\section{Abstract}

An optical coupler composed of gold cylinder arrays overlaid with a gold film was proposed for quantum well infrared photo-detector. Finite difference time-domain method was used to numerically investigate the near-field enhancement. The result showed that for the $x$-polarized light normally incident from the backside, strong electric field component perpendicular to the quantum well can be generated, which can meet the requirement of the intersubband transition and help improve the performance of QWIPs.

\section{INTRODUCTION}

Quantum well infrared photodetectors (QWIPs) have experienced rapid development in the last two decades[1]. According to the polarization selection rule of inter-subband transition[2], infrared absorption is possible only when the electric field of the incident light has a component perpendicular to the quantum well layers. In order to couple the normal incident optical wave to the quantum well active region of QWIP devices, some efficient ways[3-5] have been used.

Recently, the near field properties of the surface plasmon polariton have been employed to improve the performances of QWIPs[6-10]. In this paper, the structure composed of cylinder gold arrays overlaid with a gold film was designed as an optical coupler for quantum well infrared photo-detector. The field distribution was simulated via finite difference timedomain method. The result showed that the metallic structure can generate a nonzero electric field component parallel to the growth direction of QW, which can enhance the infrared absorption in the quantum well region.

\section{Numerical model and simulations}

The unit cell of the simulated structure is shown in fig 1. Cylinder gold arrays are integrated on the top of the quantum well region and overlaid with a gold film, the thickness of which is $\mathrm{t}=100 \mathrm{~nm}$. The period of the square-lattice gold cylinder arrays is $\mathrm{p}=6 \mu \mathrm{m}$. The height and diameter of the cylinder is $\mathrm{h}=650 \mathrm{~nm}$ and $\mathrm{d}=2.6 \mu \mathrm{m}$.

\footnotetext{
*Corresponding author: xschen@mail.sitp.ac.cn, luwei@mail.sitp.ac.cn.
}

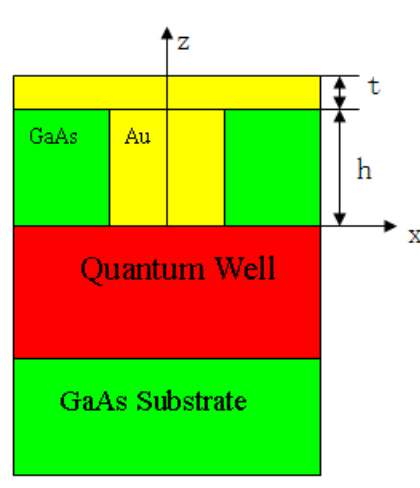

(a)

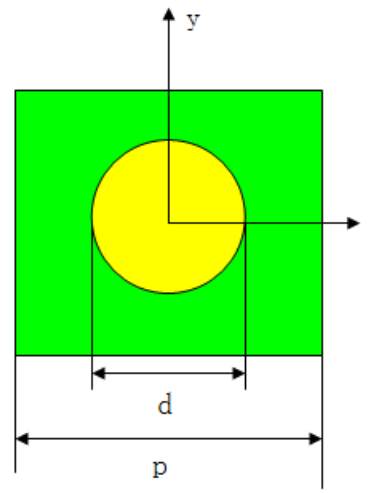

(b)
Figure 1. (Color online) Side view (a) and top view (b)of the unit cell of the simulated structure

To numerically simulate the field distribution, finite difference time-domain(FDTD) method[11] is used. Periodic boundary conditions are adopted in $\mathrm{x}$ and $\mathrm{y}$ directions and perfect matched layer(PML) conditions are imposed at the boundaries along $\mathrm{z}$ axis. The radiation source is normally incident along $\mathrm{z}$ direction and the direction of its electric field is along $\mathrm{x}$ axis. The refractive index of both the quantum well material and the GaAs substrate is set to 3.4 for simplicity. The frequency-dependent permittivity of $\mathrm{Au}$ is based on the Lorentz-Drude model[12].

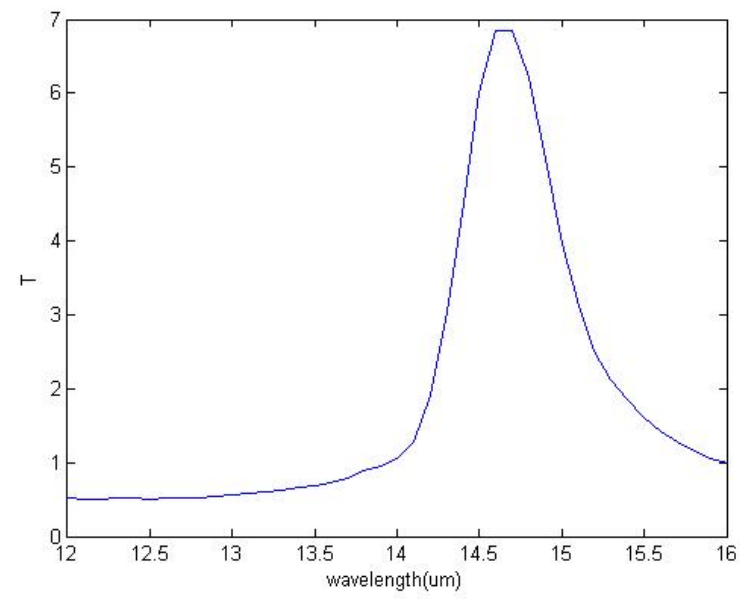

Figure 2 Enhancement spectrum at the plane locating at $\mathrm{z}=-50 \mathrm{~nm}$

We define $\mathrm{T}$ as the enhancement ratio of the averaged $|\mathrm{Ez}|^{2}$ over a unit cell in the xy plane compared with $\left|\mathrm{E}_{0}\right|^{2}$, where $\left|\mathrm{E}_{0}\right|$ is the electric field intensity of the incident light. Figure 2 
shows the value of $\mathrm{T}$ at the $\mathrm{xy}$ plane locating at $\mathrm{z}=-50 \mathrm{~nm}$ as the function of incident wavelength. A remarkable resonance peak at $14.6 \mu \mathrm{m}$ can be obviously observed in the spectrum.

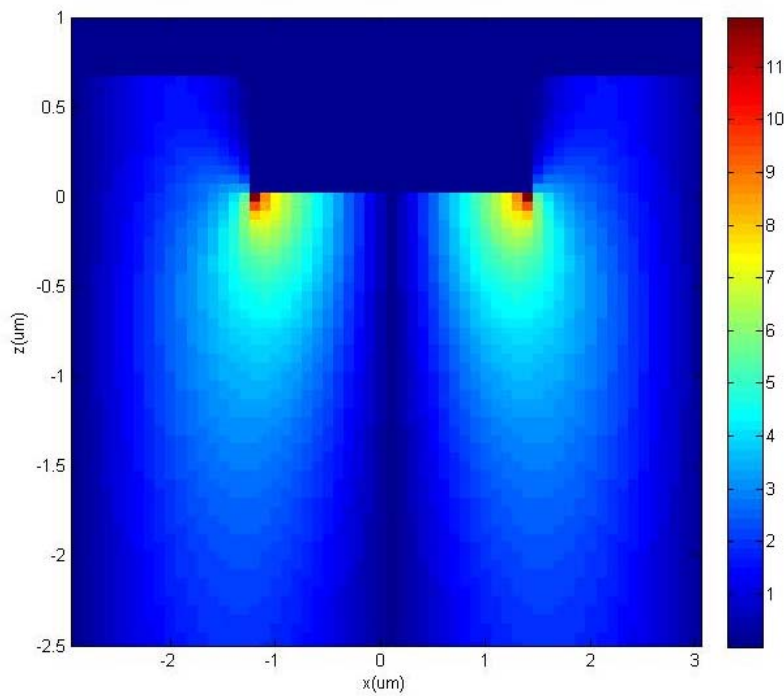

Figure 3 (Color online) $|\mathrm{Ez}|$ distribution in $\mathrm{XOZ}$ plane at $\mathrm{y}=0$

Figure 3 presents the $|\mathrm{Ez}|$ distribution in $\mathrm{XOZ}$ plane at $y=0$ for resonant wavelength $\lambda=14.6 \mu \mathrm{m}$. The metallic structure can convert the incident electromagnetic waves with oscillating electric fields in $\mathrm{x}$ direction into electric fields oscillating in the $\mathrm{z}$ direction, localized in the region below the metal surface. In the area where the enhancement is the strongest, $|\mathrm{Ez}|$ can exceed ten times as $\left|\mathrm{E}_{0}\right|$.

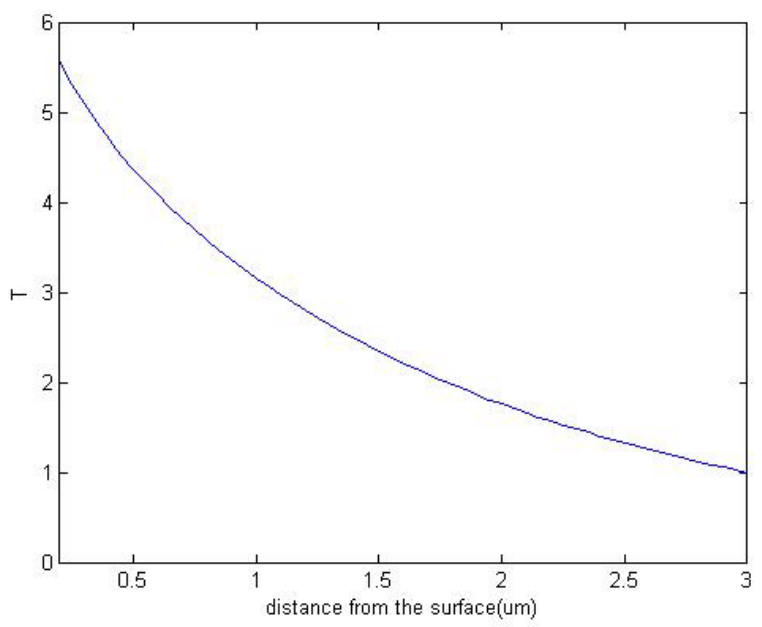

Figure 4 Enhancement as the function of the distance from the metal surface

As can be seen in Figure 4, the averaged $|\mathrm{Ez}|^{2}$ in xy plane decays exponentially with the distance from the surface, but it can still be as strong as $\left|E_{0}\right|^{2}$ at the xy plane which is $3 \mu \mathrm{m}$ below the metal surface. When the quantum well active region is located at the area $-3<\mathrm{z}<-0.2 \mu \mathrm{m}$, the coupling efficiency $\eta=\frac{\iiint_{Q W}\left|E_{z}\right|^{2} d V}{\iiint_{Q W}\left|E_{0}\right|^{2} d V}=2.5$. The photo-current is enhanced and the infrared absorption of QWIP can be improved.

\section{CONCLUSION}

The structure composed of cylinder gold arrays overlaid with a gold film was proposed as optical coupler for quantum well infrared photo-detector. Near-field effect was numerically simulated and electric field component perpendicular to the quantum well was found to be strongly localized below the metal surface, which can enhance the infrared absorption and improve the performance of QWIPs.

\section{ACKNOWLEDGEMENTS}

This work was supported in part by the State Key Program for Basic Research of China grants (2011CB922004, 2013CB632705), the National Natural Science Foundation of China grants (10990104, 61290301), the Fund of Shanghai Science and Technology Foundation grants (10JC1416100).

\section{REFERENCES}

[1] H. Schneider and H. C. Liu, Quantum Well Infrared Photodetectors Physics and Applications (Springer, 2007).

[2] B. F. Levine, "Quantum - well infrared photodetectors," J. Appl. Phys. 74, R1(1993).

[3] H.C. Liu, M. Buchanan, Z.R. Wasilewski, "How good is the polarization selection rule for intersubband transitions?”Appl. Phys. Lett. 72 (1998) 1682.

[4] M.Z. Tidrow, J.C. Chiang, S.S. Li, K. Bacher, "A high strain two-stack two-color quantum well infrared photodetector," Appl. Phys. Lett. 70 (1997) 859.

[5] C.J. Chen, K.K. Choi, W.H. Chang, D.C. Tsui, "Two-color corrugated quantum-well infrared photodetector for remote temperature sensing," Appl. Phys. Lett. 72 (1998) 7.

[6] D. Lepage and J. J. Dubowskia, "Surface plasmon assisted Photoluminescence in GaAs/AlGaAs quantum well microstructures," Appl. Phys. Lett. 91, 163106 (2007).

[7] K.-C. Shen, C.-Y. Chen, C.-F. Huang, J.-Y. Wang, Y.-C. Lu, Y.-W. Kiang, C. C. Yang, and Y.-J. Yang, "Polarization dependent coupling of surface plasmon on a one-dimensional Ag grating with an InGaN/GaN dual-quantumwell structure," Appl. Phys. Lett. 92, 013108 (2008).

[8] J. B. Khurgin and G. Sun, "Enhancement of light absorption in a quantum well by surface plasmon polariton,” Appl. Phys. Lett. 94, 191106 (2009).

[9] W. Wu, A. Bonakdar and H. Mohseni. "Plasmonic enhanced quantum well infrared photodetector with high detectivity," Appl. Phys. Lett. 96, 161107 (2010)

[10] S.-Q Zhai, J.-Q Liu, F-Q Liu and Z-G Wang. "A normal incident quantum cascade detector enhanced by surface plasmons," Appl. Phys. Lett. 100, 181104 (2012)

[11] A. Taflove and S. C. Hagness, Computational Electrodynamics: The Finite Difference Time-Domain Method, $2^{\text {nd }}$ ed. (Artech House, 2000).

[12] A. D. Rakic', A. B. Djurišic', J. M. Elazar, and M. L. Majewski, “Optical properties of metallic films for vertical-cavity optoelectronic devices," Appl. Opt. 44, 5271-5283 (1998). 